\title{
Case Study: Leading Change across Two Sites: Introduction of a New Documentation System
}

Andrea R. Walker, RN, BSN, MSN

General Nurse Educator, Providence Health Care

Vancouver, BC

\begin{abstract}
Leading change initiatives across multiple geographic locations has become increasingly frequent among nursing leaders as hospitals merge to form more effective and powerful organizations. This case study illustrates how strong nursing leadership, in conjunction with a transformational change approach, led to the successful implementation of a new documentation system at two hospitals within one organization. The project process is described in detail, including a discussion of lessons learned, and recommendations are provided for the leadership of future multi-site projects.
\end{abstract}

Hospital mergers are on the rise in Canada (Markham and Lomas 1995) and so, therefore, is the need to lead across multiple geographic locations. This form of restructuring is accompanied by other complex and rapid changes in healthcare (Taccetta-Chapnick 1996). Because of the need to manage change in such a challenging environment, the Canadian Nurses Association (2005) has called for greater nursing leadership.

MacLaren (2002), a director of transitional services in Southern California, claimed that the most common reason to lead or manage across more than one hospital site is a merger. Jordan and Stuart (2000), both high-profile consultants working in the healthcare sector, provided several reasons why two or more hospitals may merge, 
such as achieving cost savings, gaining increased power or leverage, fulfilling a strategic vision, improving access to services or providing an increased continuum of services, attracting specialized staff and/or research funding and responding to pressures from key stakeholders. After conducting a review of literature on multiple hospital arrangements from Canada and the United States, Markham and Lomas (1995) added that combining forces leads to improved buying power by ordering supplies in larger quantities, better recruitment of human resources owing to more attractive specialties, and a potential decrease in wait list times due to improved access to care. When multiple hospitals merge they can easily share best practices and learn from one another's mistakes, developing optimal policies and procedures to guide practice (Jordan and Stuart 2000).

However, despite the many advantages identified for hospital mergers, many disadvantages have also been documented. There is a period of increased costs with the initial amalgamation; a lack of easy access to services offered at only one site and the attendant need for patient transportation; exacerbated levels of staff insecurity, stress, anxiety and resistance; and erosion of hospital loyalties (Markham and Lomas 1995). Although hospitals have the potential to save money with shared managers and leaders (MacLaren 2002), leading across more than one location may be one of the most difficult challenges resulting from mergers.

\section{Background}

Providence Health Care (PHC) is the largest Catholic hospital organization in Canada. It operates seven facilities situated in Vancouver, British Columbia. These include three hospitals delivering acute care, residential, and rehabilitation services, three residential facilities that focus on intermediate and long-term care for the elderly, and a hospice. In the early 1990s, regionalization concepts were discussed by the nuns who operated the various healthcare facilities in the Lower Mainland. They needed to create a plan to protect Catholic healthcare within a regionalized system and to ensure that leadership for Catholic healthcare would continue despite the dwindling number of sisters. As a result, three congregations merged to form CHARA Health Care Society. PHC was formed through the consolidation of CHARA, Holy Family Hospital and St. Paul's Hospital in 1997, and became a single legal entity in 2000 .

\section{The Project}

The project task was to implement a new documentation system at St. Paul's Hospital (SPH) and Mount St. Joseph Hospital (MSJ). SPH is a 500-bed acute care, academic and research hospital, whereas MSJ is a smaller 208-bed, acute and extended care facility. The new documentation system was to be introduced to the medical and surgical units, for a total of 18 patient units (14 at SPH and 4 at MSJ). 
One of the main reasons propelling the need to change the documentation system at PHC was to prepare for the imminent arrival of electronic documentation. The previous system allowed considerable variation in the terminology used to describe similar assessments and encouraged the use of narrative notes. Preparing for electronic documentation required the introduction of common terminology and a decrease in the amount of redundant narrative entries in patient records. Standardizing the documentation forms would also assist in a smoother transition when education was required for all nurses on how to shift from paper forms to the electronic version.

Another goal of the project was to improve interdisciplinary communication. Previous documentation practices discouraged the use of an integrated patient record by having each discipline document separately from the others. The new system would facilitate interdisciplinary communication and patient planning.

Other perceived benefits of introducing a new documentation system were to improve the standard of care and patient safety. These were anticipated because the new forms could be used as a guideline for assessments and as prompts to assess for patient safety. The introduction of standardized assessments would also assist in data collection for research purposes and the ability to track trends more easily.

The new system introduced three new forms: (a) the patient biography (used to collect detailed patient information on admission and to guide the process of referrals to allied healthcare providers), (b) the 24-hour patient care flowsheet (used by nursing to document vital signs, assessments, treatments and interventions) and, lastly, (c) the interdisciplinary progress notes (used to facilitate entries on patient progress and planning by all disciplines).

\section{The Process}

Strong nursing leadership, in conjunction with a transformational change approach, led to the successful implementation of the new documentation system across both sites. Kotter's (1995) recommended strategies to implement a complex change initiative guided the process, including establishing a sense of urgency for the change, forming a powerful coalition of a large group of leaders, creating and communicating the vision, empowering others to get involved, celebrating accomplishments and incorporating the change into the organization's culture.

An external consultant assessed the need to change the current documentation system by canvassing staff nurses and scanning the practices of other organizations. Concurrently, documentation practices were scrutinized and researched to develop the beginnings of the new forms and guidelines. An Interdisciplinary Documentation Committee, with representation from all sites and disciplines, was 
formed in November 2003 to oversee documentation issues, concerns and practices. This group developed principles to guide the development and subsequent revisions of the new documentation system. A Nursing Work Group was also established (active from January 2004 to January 2005), including membership from managers, clinical nurse leaders (CNLs), nurse educators (NEs) and nurses, and representing SPH and MSJ. This group was responsible for the refining and testing of the new forms.

A trial of the new forms was conducted on a medical and on a surgical unit at SPH in November 2004. Prior to the trial, the nurses were educated on how to use forms, why the forms were changing and how they would be supported through the change process. Three nurses from the trial areas were provided with special education on the management of change and were used as coaches for three weeks post-implementation. Their primary role was to assist the nurses in using the forms, but they also collected data on staff satisfaction, recommendations for revisions and the proper use of the forms, resulting in three subsequent form revisions.

To prepare the remainder of the nurses at SPH for the upcoming changes and to communicate the vision, an awareness campaign was launched in the fall of 2005. The project team attended staff meetings, made posters and spoke to leadership about the upcoming changes. Meetings were conducted with the leadership from each unit to gain support for the project and to address needs particular to each unit and its staff. CNLs and NEs received extensive education on the new forms, how to manage change and how to support their staff through this transition. They were also provided with clear role expectations. In January 2006, nurses attended education sessions in which they learned why the documentation system was changing, how to use the new forms and how they would be supported through the process.

The new documentation system was implemented on 14 medical and surgical units at SPH in February 2006. Coaches (trained nurses) were recruited from a variety of units (including one from MSJ) to encourage, provide feedback and educate their colleagues on how to use the new forms. They also audited charts to assess the proper use of the forms and were given a clear structure to assist with problem solving. Coach support was available for three weeks.

A new NE from MSJ, in collaboration with a project leader from SPH, implemented the new system at MSJ. The strategies used for the roll-out at SPH were also used at MSJ, including an awareness campaign, education on managing change and role expectations for the unit leadership, education for staff nurses and the recruitment and training of nurses to coach. The new documentation was implemented on one surgical and three medical units in May 2006, with coaching 
support available for two weeks. At this site, CNLs were also utilized as coaches, along with the MSJ nurse who coached at the SPH implementation.

Chart audits were conducted at both sites three months after their respective implementation dates, and a meeting was held with all NEs to follow up with any areas that required further tweaking of the system or additional education. A plan to have audits continue was also discussed. Implementation of the system at both sites was successful, and the nurses were recognized for their hard work.

\section{Lessons Learned and Recommendations for Future Multi-Site Change Initiatives}

A well-designed project plan and effective leadership from the project team were of central importance to the success of the project at both sites. In addition, support from senior leadership to ensure that there were appropriate resources was fundamental to achieving project results. Forming a powerful coalition of strong nursing leaders to propel this initiative forward was of paramount importance, and this was accomplished by involving leaders from both sites in developing a shared vision and in the planning and creation of the new forms.

It is recommended that a thorough assessment of the culture of each site be conducted prior to implementing change across more than one location. In the assessment and planning phases of the project, the consultant spent more time investigating the culture and readiness for change at the SPH site and may have neglected to assess the culture of MSJ properly. With the merger of any facilities, work must be done to remove feelings of one "taking over" the other or being the "bully." Some of these feelings have persisted, nearly 10 years after the merger. Therefore, sufficient time and energy need to be invested in the amalgamation of all sites, demonstrating equality of power as well as listening to and validating concerns from each site. Such attention may assist with the implementation of future projects across sites.

The successful implementation of the new documentation at MSJ can be attributed to having the MSJ NE seen as the project leader. This strategy facilitated the ownership of the project by the nurses at MSJ. However, in this instance, the positive effect of this strategy may have been somewhat more difficult to achieve, as the NE was relatively new to MSJ and may not have established the necessary relationships with the staff. In addition, the MSJ NE left the organization shortly after the implementation phase of the project. This departure necessitated a transfer of leadership for the support and evaluation phases, resulting in some confusion. It is recommended that project leaders at each implementation site be present at that facility for an extended period both prior to and after implementation of the initiative. Their presence could contribute to nurses' development of trust in the 
leader, provide the leader with a history of how past projects were managed and assist with the management of any followup issues.

Using nurses as coaches at both locations was crucial to achieving buy-in at both sites. The coaches had a deep understanding of the real issues on the units and had already achieved credibility with their colleagues. It was also extremely beneficial to use a nurse from MSJ as a coach for the SPH roll-out. This facilitated the awareness campaign at MSJ by direct and influential contact with MSJ nurses. Owing to a shortage of nurses, CNLs at MSJ were used as coaches. These leaders were encouraged to take more ownership over the success of the project and therefore developed an enhanced awareness of details, giving them greater leverage in problem solving. The combination of using both nurses and leadership as coaches is recommended.

Nurse shortages at both sites contributed to the nurses' stress while learning the new documentation system. If resources are available, nurses should be adequately supported by providing additional staff on the units for the first few weeks during the initial transition. Such support would allow each nurse sufficient time and energy to learn the new system and adapt to the change.

It may be beneficial to test the system at all sites, as a trial could facilitate buyin from the nurses and contribute to their ownership of the project. However, because the previous documentation systems were different at each site, and because MSJ's system was closer to the new version, the decision was made to test the new system only at SPH. With the next move to electronic documentation, a trial at both sites, together with combined education, is recommended.

\section{Conclusion}

The implementation of a complex change initiative across multiple sites is a challenging task that can be accomplished using strong and effective leadership. The experience of implementing a new documentation system across two sites suggests that while it can be done successfully, there is always latitude for strengthening strategies. Although further research is needed to document the relationship between the described approaches and successfully leading change across multiple locations, this case study provides nursing leaders with a resource when faced with a similar challenge. 


\section{References}

Canadian Nurses Association. 2005. "Nursing Leadership in a Changing World." Nursing Now: Issues and Trends in Canadian Nursing 18: 1-6.

Jordan, M. and N. Stuart. 2000. "Hospital Mergers: Why Some Fail and Others Succeed." Hospital Quarterly 3(2): 41-45.

Kotter, J.P. 1995. “Leading Change: Why Transformation Efforts Fail.” Harvard Business Review 73(2): 59-67.

MacLaren, E. 2002. “One for All: Combining Management across Hospitals.” Nursing Management 33(7): 32-33.

Markham, B. and J. Lomas. 1995. "Review of the Multi-Hospital Arrangements Literature: Benefits, Disadvantages and Lessons for Implementation." Healthcare Management Forum 8(3): 24-35.

Taccetta-Chapnick, M. 1996. “Transformational Leadership.” Nursing Administration Quarterly 21: $60-66$.

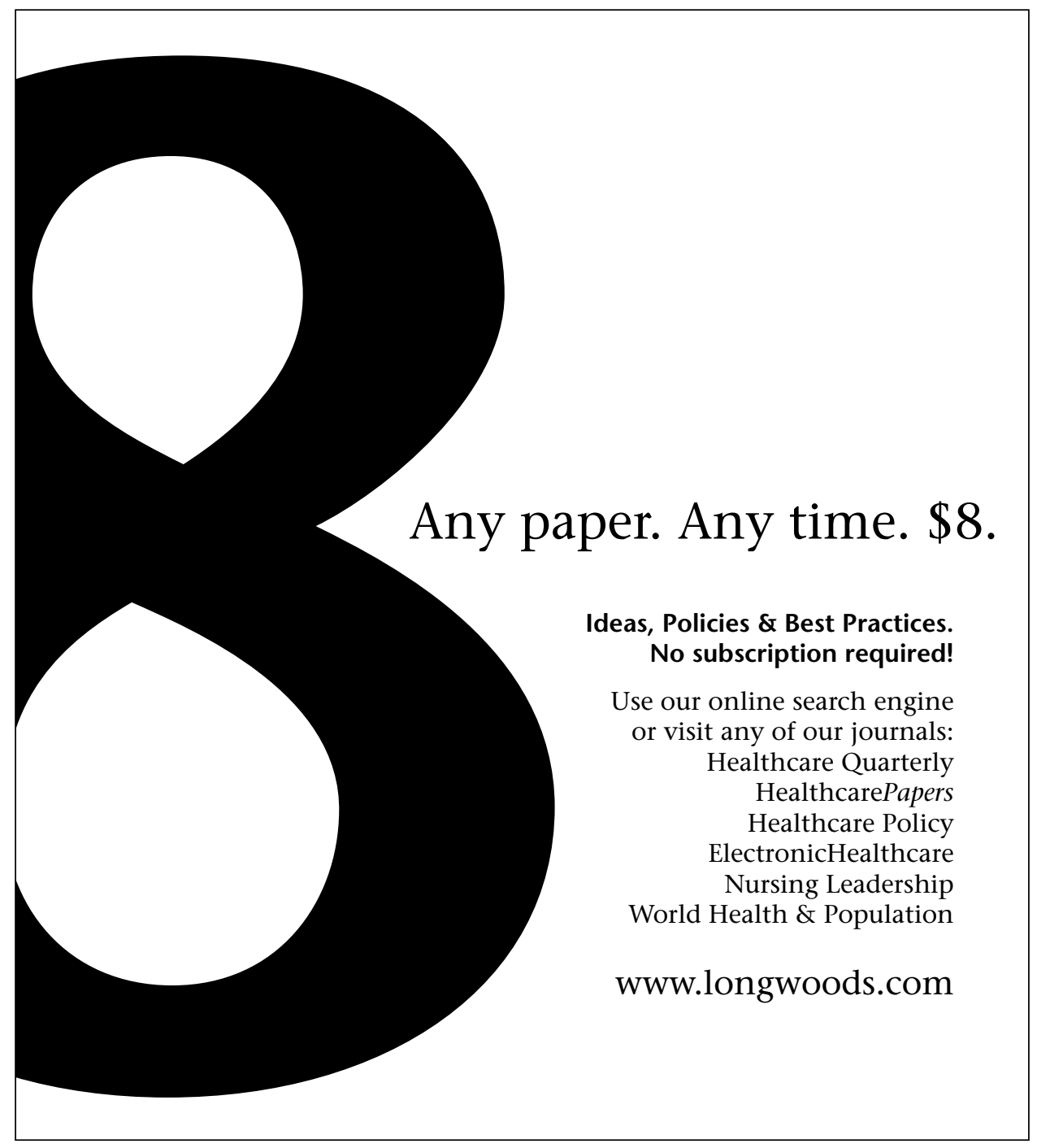

